\title{
Impact of Shunt reactor to DFIG Connected to Series- Compensated Power System on Sub-synchronous Oscillation Characteristics
}

\author{
Yang $\mathrm{WU}^{1}$, Kunyu $\mathrm{LIAO}^{1}$, Xiangning $\mathrm{XIAO}^{1}$,Chao $\mathrm{LUO}^{2}$ \\ ${ }^{1}$ School of Electrical \& Electronic Engineering, North China Electric Power University, Beijing 102206, China \\ ${ }^{2}$ Southern Power Grid Science Research Institute, Guangzhou 510080, China
}

\begin{abstract}
The shunt reactor will affect the sub synchronous oscillation characteristics of the wind power system, and there is no paper that analyse this aspect. To this end, in the DFIG via series compensation transmission power system, use impedance scanning method to measure the total equivalent impedance of system side and DFIG side, the mechanism of the influence of the installation position and capacity of the shunt reactor on the sub synchronous oscillation characteristic of the system is analysed. The analysis results show that the larger capacity of shunt reactor, equivalent impedance effect is larger, the installation position that is very near the fan can reduce the resonant frequency and increase the resistance of resonance point, being far from the fan will increase the resonant frequency and the absolute value of the negative resistance in the resonance points, besides, the effect of parallel capacitor on system impedance is very small. Finally, the correctness of the theoretical analysis is verified by the time-domain simulation.
\end{abstract}

\section{$1 \quad$ INTRODUCTION}

Series capacitor compensation can effectively and economically improve the transmission distance and improve the stability of the power system. It is also one of the main transmission measures for the long-distance transmission of wind farms. But the series compensation also brings the risk of sub-synchronous oscillation to the power grid. In 2009, a sub-synchronous resonance accident caused by a series of capacitors occurred in a wind field in Texas, USA [1].

The sub-synchronous oscillation about wind turbine generator including effect of wind turbine shafting and fixed series compensation between torsional interaction , induction generator effect, sub-synchronous control interaction. The papers [2-5] use impedance scanning method to analyze the by DFIG series compensation grid sub synchronous oscillation mechanism, pointed out that the reason of the oscillation is that DFIG may appear negative resistance in the sub-synchronous frequency.

Shunt reactor, which is widely used in static reactive compensation device, has not been mentioned in the literature. However, when the system structure changes, the frequency of sub-synchronous oscillation caused by DFIG will also change, so it is necessary to study the influence of installation location and capacity of shunt reactor on the sub-synchronous oscillation characteristics of the system.
Firstly, from the perspective of the impedance analysis of DFIG series compensation mechanism of feeding sub-synchronous oscillation, pointed out that the reason for the negative resistance at the resonance frequency of the formation with the DFIG system.And then the total equivalent impedance are measured and the system side of DFIG with frequency scanning method, analyzed the relationship between the location and capacity of shunt reactor device size and subsynchronous oscillation characteristics change.

\section{SYSTEM MODELING}

Based on the local topology of a wind power supply system in North China, the equivalent fan grid model, as shown in Figure 1, is established in this paper. As shown in Figure 1, where DFIG is the rated capacity of wind farm 220MVA; L1, L2 and L3 are expressed as 35kV collective line and $220 \mathrm{kV}$ transmission line and $500 \mathrm{kV}$ transmission line; T1, T2 and T3 represent $0.69 \mathrm{kV} / 35 \mathrm{kV}$ box change, $35 \mathrm{kV} / 220 \mathrm{kV}$ boost change and $220 \mathrm{kV} / 500 \mathrm{kV}$ boost change, respectively.; $X_{\mathrm{C}}$ represents a fixed series compensation. I, II, III, IV said the shunt reactors are installed respectively, where $\mathrm{I}$ is the $35 \mathrm{kV}$ side of the transformer T1, II line midpoint collective line L1, III for the $220 \mathrm{kV}$ side of the transformer of T2, IV for the $220 \mathrm{kV}$ side of the transformer T3. 


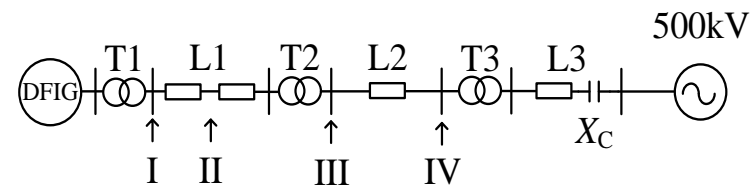

Figure 1: The system diagram of a DFIG via a series complementary grid

\section{MECHANISM ANALYSIS}

For the research system, the equivalent circuit under the sub-synchronous frequency is shown in Figure 2.

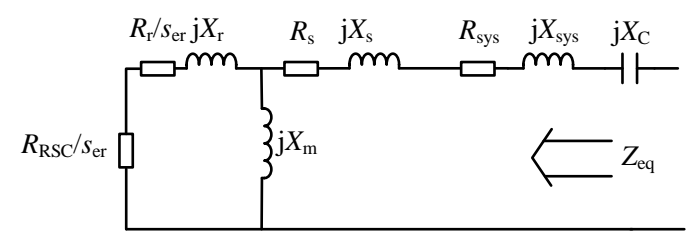

Figure2: Sub-synchronous frequency equivalent circuit

$s_{\mathrm{er}}$ is the slip; $R_{\mathrm{r}}, R_{\mathrm{S}}$ for the rotor resistance, stator resistance of DFIG, $R_{\mathrm{RSC}}$ for the rotor side converter of equivalent resistance; $X_{\mathrm{r}}, X_{\mathrm{s}}, X_{\mathrm{m}}$ are respectively rotor leakage reactance, stator leakage reactance, excitation reactance in sub-synchronous frequency; $R_{\text {sys }}, X_{\text {sys }}$ respectively, the system side resistance the synchronous frequency contains the line reactance and the transformer leakage reactance of the system side.

The sub-synchronous resonance frequency of the DFIG supply system is as follows:

$$
f_{\text {er }}=f_{0} \sqrt{\frac{X_{\mathrm{C}}}{X_{\Sigma}}}
$$

In the formula (1), $f_{\text {er }}$ is the sub-synchronous resonance frequency, $f_{0}$ frequency synchronization, $X_{\mathrm{C}}$ series capacitor capacitance, $X_{\Sigma}$ is total equivalent inductance of DFIG and the system, because the $X_{\mathrm{C}}<X_{\Sigma}$, so $f_{\text {er }}<f_{0}$.

Under the sub-synchronous frequency $s_{\mathrm{er}}$ is:

$$
s_{\text {er }}=\left(f_{\text {er }}-f_{\mathrm{r}}\right) / f_{\text {er }}=1-f_{\mathrm{r}} / f_{\text {er }}
$$

$f_{\mathrm{r}}$ is the rotor speed and frequency of doubly fed induction generator, and is related to wind speed. When the wind speed remains constant, $f_{\mathrm{r}}$ can be regarded as a constant value due to the change of rotor speed in short time oscillation, and $f_{\mathrm{er}}<f_{\mathrm{r}}$ is generally $s_{\mathrm{er}}<0$.

$$
R_{\text {req }}=\frac{R_{\mathrm{r}}+R_{\mathrm{RSC}}}{S_{\mathrm{er}}}
$$

When the rotor side ring dynamic is ignored, the value of $R_{\mathrm{RSC}}$ is approximately equal to the inner loop gain of the rotor current [6], so there is $R_{\text {req }}<0$ at the subsynchronous frequency. From the (2) formula, as the $f_{\mathrm{er}}$ increases, the $s_{\mathrm{er}}$ decreases, so the absolute value of the negative resistance is getting bigger and bigger. When the absolute value of $R_{\text {req }}$ at a resonance frequency is greater than that of the stator side and the side of the system, the total equivalent resistance is negative. In this case, the negative damping divergent oscillation will occur.

\section{IMPEDANCE SCANNING}

\subsection{INFLUENCE OF SHUNT REACTOR INSTALLATION POSITION}

In this paper, a parallel current source method is used to conduct impedance scanning analysis of the system shown in Fig. 1 without a shunt reactor. The initial wind speed of the DFIG is $6.5 \mathrm{~m} / \mathrm{s}$, and the series compensation degree of the circuit is $25 \%$. The installation point of shunt reactor is the four points of I, II, III and IV in Figure 1, which represent the distance relation of the distance between the shunt reactor and the DFIG respectively.

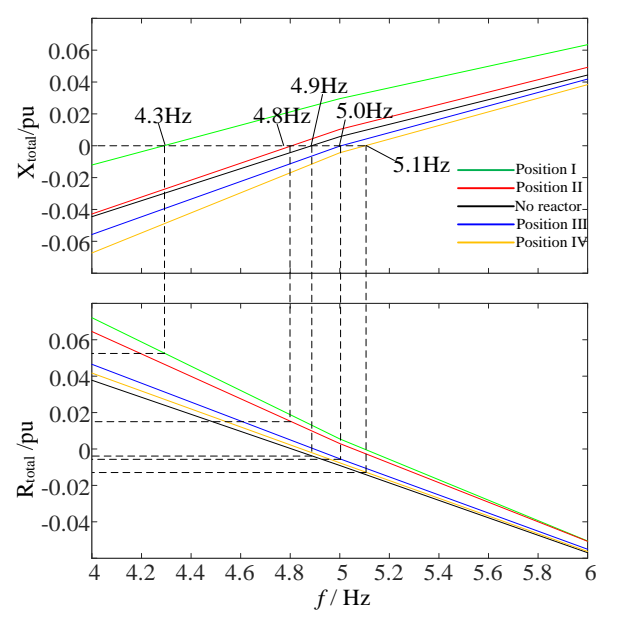

Figure 3: Impedance scanning results of parallel reactor with different installation position

First, the shunt reactor with compensation capacity of 20MVar is selected for frequency scanning analysis at each installation point. The frequency sweep curve of the total equivalent resistance $R_{\text {total }}$ and the equivalent reactance $X_{\text {total }}$ installed at different locations is shown in Figure 3.

From Figure 3, When the reactive power compensation is not installed, the equivalent reactance of the system is equal to 0 at $4.9 \mathrm{~Hz}$, that is, the resonance occurs at this point, and the equivalent resistance of the system is less than 0 , so the system will have a negative damping divergent oscillation at $4.9 \mathrm{~Hz}$. We can see when the shunt reactor is installed, the equivalent resistance curve is shifted upwards compared with the initial system. Located on I and II, which is from the fan is very near, the total equivalent reactance of zero crossing to the left, the resonant frequency decreases and the resonance frequency from the original system of $4.9 \mathrm{~Hz}$ is reduced to $4.3 \mathrm{~Hz}$ and $4.8 \mathrm{~Hz}$, and the resonance point corresponding to the $R_{\text {total }}>0$, so the system will not happen sub-synchronous oscillation; installed in the position of III and IV, which is far away from the fan, the resonant frequency of the system increases to $5.0 \mathrm{~Hz}$, while the equivalent resistance curve near the resonance frequency of the resonant frequency has been on the move, The absolute value of the $R_{\text {total }}$ (less than 0 ) at the resonant point is larger than the absolute value of the uninstalled, damping decreases, increased the sub- 
synchronous oscillation in the two the installation position. It can be seen that the farther the installation position of shunt reactor is from the fan, the greater resonant frequency of the system is.

\subsection{INFLUENCE OF CAPACITY SIZE OF SHUNT REACTOR}

Since the shunt reactor is installed at I or II, it will improve the sub-synchronous oscillation characteristics of the system. When installed at III or IV, it will aggravate the system oscillation. Therefore, taking I and III as an example, the influence of the compensation capacity on the system equivalent impedance is analysed. The shunt reactor with capacity of 10MVar, 20MVar and $30 \mathrm{MVar}$ is installed at the installation point I and III respectively. As shown in Figure 4, the resonant frequency of the system gradually decreases with the increase of the shunt reactor capacity, and the $R_{\text {total }}$ of the system increases gradually at the resonant point, which is more than 0 . Therefore, when the shunt reactor installation point is close to the fan, the larger the compensation capacity is, the larger the $R_{\text {total }}$ of the sub synchronous resonance point, the stronger the system damping, which reduces the risk of SSO.

From Figure 5, we can see that at the installation point III, the resonant frequency of the system increases with the increase of the shunt reactor capacity. Although the $R_{\text {total }}$ near the resonant frequency increases gradually, the absolute value of the negative resistance at the resonant point is bigger and bigger. Therefore, when the shunt reactor installation point is far away from the fan, the greater the compensation capacity and the weaker the damping of the system, the more easy to increase the sub synchronous oscillation of the system.

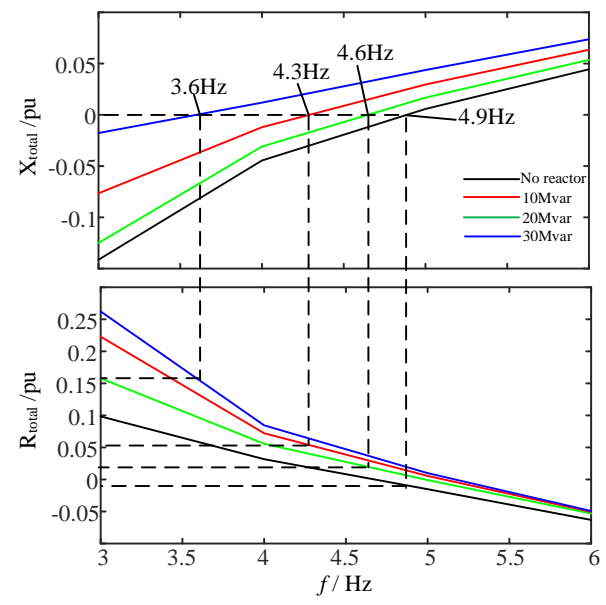

Figure 4: Impedance scanning results of different compensation capacity at the installation point I

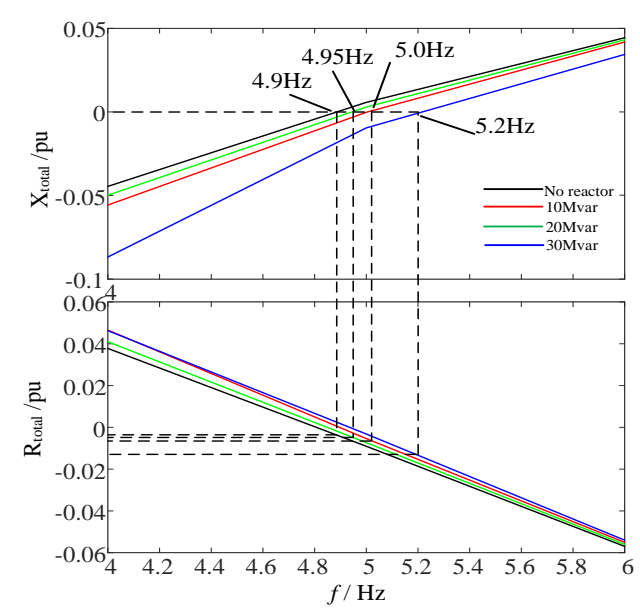

Figure 5: Impedance scanning results of different compensation capacity at the installation point III

From the above analysis, the sub synchronous resonance frequency on the initial system for $f_{S}$ shunt reactor system sub-synchronous resonance frequency of $f_{\mathrm{SX}}$, when $f_{S}>f_{\mathrm{SX}}$, the shunt reactor will increase the total resistance of the resonance point, so as to improve subsynchronous oscillation of the system; when $f_{S}<f_{\mathrm{SX}}$, the shunt reactor will reduce the total equivalent resistance of the resonance point, thus exacerbating the SSO.

\section{VERIFICATION BY TIME DOMAIN SIMULATION}

Use time-domain simulation to preliminary analyse subsynchronous oscillations of the system. In this case, no shunt reactor is connected to the system, and a series compensating capacitor is put into the system for exciting the sub-synchronous oscillation at 5 seconds. As shown in Figure 7, after inputting series compensation, the system quickly produce divergent oscillation. We can find that the frequency of sub-synchronous oscillation is $4.9 \mathrm{~Hz}$, so the simulation results and the previous impedance scanning results correspond with each other.

As shown in Figure 6, figure 6 (a) is a comparison chart of output power of DFIG when 20MVar shunt reactor is installed in the position I and II, figure $6(\mathrm{~b})$ is a comparison chart of output power of DFIG when 20MVar shunt reactor is installed in the position III and IV. When the series compensation is put into at 5 seconds the oscillation is convergent at position I and II, and the installation position nearer from the DFIG, the convergence speed is faster; installed in the III and IV, oscillations are more serious than the original system, and the installation position farer from the DFIG, the divergence speed is faster. This is matched with the results of the previous analysis. 


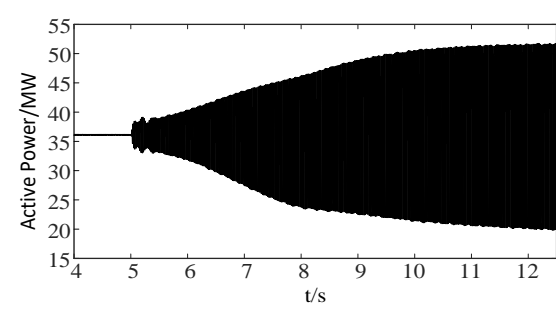

(a) Output active power of DFIG

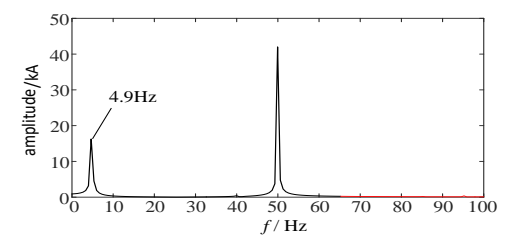

(b) Spectrum analysis of A phase current

Figure 6: Time domain simulation results of initial system

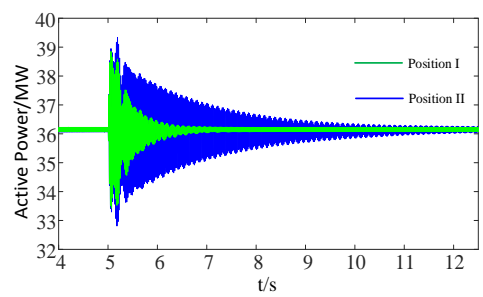

(a) Position I and Position II

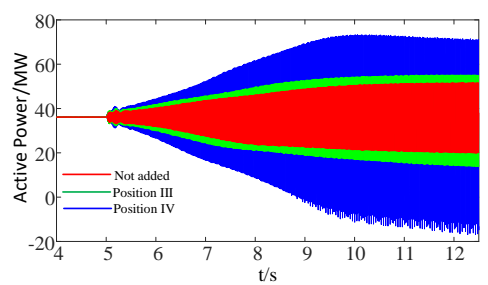

(b) Position III and Position IV

Figure 7: The comparison diagram of the output power of the DFIG with different shunt reactor installation position

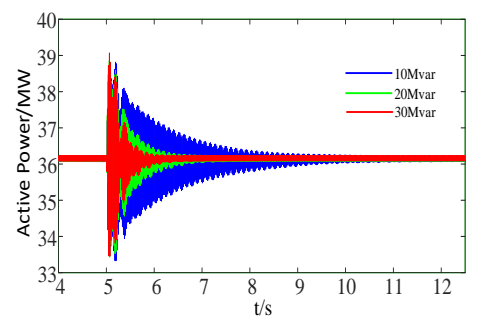

(a) Position I

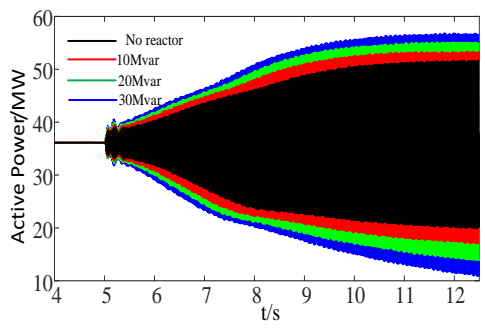

(b) Position III

Figure 9: The comparison diagram of the output power of the DFIG with different shunt reactor compensation capacity
The time domain simulation results of different compensation capacity of shunt reactors are shown in Figure 7. In figure 7 (a), shunt reactor is installed in position I, in figure 7 (b), shunt reactor is installed on position III. It is known that at the position I, with the increase of the compensation capacity of the shunt reactor, the convergence speed of the system oscillates faster, while at the position III, the trend of change is opposite to that of I, which is consistent with the conclusion obtained from the impedance scan results.

Figure 8 shows the system when the sub-synchronous oscillation occurs, put 20MVar shunt reactors at the position $I$ in $7 \mathrm{~s}$, we can find that the reactor makes the system oscillation from divergence to convergence. At the same time, it is not difficult to analyse that when the system is in stable operation, if the shunt reactors that have been put into operation are cut off in places close to the DFIG, such as the positions I and II, or the positions IV and IV are further away from the DFIG Shunt reactor, it may make the system to stimulate sub-synchronous oscillation. At the same time, it can be seen from the above analysis that the closer the shunt reactor is installed to the fan, the greater the compensation capacity, the more the risk of sub-synchronous oscillation in the system can be reduced.

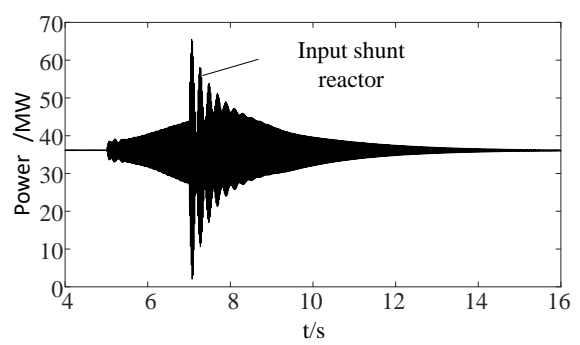

Figure 8: Influence of inputting shunt reactor on subsynchronous oscillation of the system

\section{CONCLUSION}

In this paper, the mechanism of sub-synchronous oscillation in the system with DFIG is analysed, and the reasons for the negative damping divergent oscillation are pointed out. Based on the actual system, the influence of installation position and capacity of parallel shunt reactors and shunt capacitors on the equivalent impedance of the system is analysed based on the actual system by frequency sweep and verified by time-domain simulation. The main conclusions are as follows: When shunt reactors are installed close to the wind turbine, the sub-synchronous resonance frequency will be reduced, the equivalent resistance of sub-synchronous resonance point will be improved and the sub-synchronous oscillation characteristics of the system will be improved. The larger the compensation capacity, the better the effect obviously, when the installation point is far away from the fan, the sub-synchronous resonance frequency of the system will increase, and the system equivalent resistance of the resonance point will be smaller than without shunt reactor, this will exacerbate the system sub-synchronous oscillation. 
Aiming at the sub-synchronous oscillation problem of DFIG by series transmission system, the research work in this paper can provide theoretical guidance for the installation location, compensation capacity and switching strategy of shunt reactors in actual wind power grid connection project.

\section{REFERENCES}

1. Kundur, Prabha. "Power system stability and control, "China Electric Power Press ,2001.

2. R.Li, Y.Lu, H.L.Liu, "Mechanism Analysis on Subsynchronous Oscillation Caused by Grid-Integration of Doubly Fed Wind Power Generation System via Series Compensation (in Chinese),"Power system technology, Beijing, vol.37, No.11, 2013, pp.30733079.

3. Suriyaarachchi D H R, Annakkage U D, Karawita C, et al, "A Procedure to Study Sub-Synchronous Interactions in Wind Integrated Power Systems," IEEE Transactions on Power Systems, vol.28, No.1, 2013, pp:377-384.

4. B.Zhao, "Study on Sub-synchronous Oscillation Characteristics and Suppression Strategy of Doubly Fed Wind Farm Grid Connection System (in Chinese)," Chong Qing University, 2015.

5. L.Wang, X.R.Xie, Q.R.Jiang, et al. "Analysis and Suppression of Sub-synchronous Resonance in Large Scale Doubly-Fed Wind Farm (in Chinese)," Automation of Electric Power System, vol.38, No.22, 2014, pp: 26-31.

6. Shafiu A, Anaya-Lara O, Bathurst $G$, et al. "Aggregated Wind Turbine Models for Power System Dynamic Studies," Wind Engineering, vol.30, No.3, 2006, pp: 171-185. 